\title{
A case of homicidal intraoral gunshot and review of the literature
}

\author{
Sandra Berens • Thomas Ketterer • \\ Beat P. Kneubuehl • Michael J. Thali • \\ Steffen Ross $\cdot$ Stephan A. Bolliger
}

Accepted: 21 October 2010/Published online: 13 November 2010

(C) Springer Science+Business Media, LLC 2010

\begin{abstract}
Determination of the manner of death in case of intraoral firearm wounds can be a challenge, especially if the circumstances of the incident are unclear and crime scene investigation is inadequate. It is a well-known fact that the mouth is one of the selected sites for suicide with firearms. Homicidal shooting through the mouth is said to be rare, but does occur, and can be mistaken for a suicide. For discrimination between suicide and homicide in cases of intraoral firearm wounds, some useful points are the site of entry wound, the direction of the internal bullet path, the range of fire and the circumstances of death. We demonstrate these points in a case of a homicidal gunshot to the mouth assessed by both classical autopsy and post-mortem CT (PMCT).
\end{abstract}

Keywords Intraoral gunshot $\cdot$ Homicide $\cdot$ Suicide . Virtopsy, post-mortem CT

\section{Introduction}

The typical entrance site in firearm suicides are the temple, the forehead, the left chest, and in $20 \%$ of cases the mouth [1]. Determination of the manner of death in the case of intraoral firearm wounds can be difficult, especially if death

S. Berens $(\bowtie) \cdot$ T. Ketterer - B. P. Kneubuehl ·

M. J. Thali · S. Ross - S. A. Bolliger

Department of Forensic Medicine, Institute of Forensic

Medicine, University of Bern, Buehlstrasse 20,

3012 Bern, Switzerland

e-mail: sandra.berens@irm.unibe.ch scene investigation is unclear and/or inadequate [2]. Contact intraoral gunshot wounds are much more frequently associated with suicide than with homicide [3]. Homicide cases of intraoral gunshot injuries have been described rarely in literature [2-4].

In the following case, we present an extraordinary case of homicidal gunshot to the mouth with classic autopsy and post-mortem computed tomography (PMCT).

\section{Case history}

\section{Circumstances}

The body of a 63-year-old male was found on a slope, underneath a bus station, near a main road. The body was laying head-down, 20-30 meters under the road level. The first inspection at the scene revealed a gunshot wound to the mouth with destruction of the upper incisors and injuries of the gums and internal surface of the upper lips. There was no detection of a firearm exit wound. Gunpowder residues were found on the inner surface of the lips. No weapon was found at the scene. Investigators strongly suspected that the body could be a victim of a homicide by a near contact gunshot to the mouth.

Two days before, the body of a 58-year-old male was found with a contact gunshot to the right temple in a car registered to the above-mentioned 63-year-old. The weapon found in this car was a pistol Walther PP (Manurhin), calibre $7.65 \mathrm{~mm}$ Browning (32 Auto). In addition, two spent cartridges were found in the car, but the body of the 58-year-old showed only one gunshot wound. Furthermore, blood traces and tooth remains were detected in the car which could be attributed to the 63 -year-old by DNA analysis. 


\section{Technical data}

The body of the 63-year-old was transported to the Centre of Forensic Imaging at the University of Bern and scanned in an artefact-free body bag (Rudolph Egli AG, Bern, Switzerland). Whole body post-mortem CT (PMCT) was performed using a helical 6-slice CT (Somatom 6, Siemens Medical Solutions, Forchheim, Germany). Primary image review and 3D reconstructions of the skull and neck were carried out on a CT workstation (Leonardo, Siemens Medical Solutions, Germany).

\section{Findings}

\section{PMCT}

CT examination of the skull and neck revealed a contact entrance gunshot wound to the middle of the maxilla (Fig. 1) with loss of the upper incisors (teeth 11 and 21). The wound track passed through the left massa lateralis of the atlas (Fig. 2). Bullet remains were seen in the paramedian neck musculature on the left at the $\mathrm{C} 1 / 2$ level. There was no exit wound. Intraspinal bone fragments and a lesion of the medulla at the level of $\mathrm{C} 1$ were seen. The bullet path showed a direction from anterior to posterior, from median to paramedian left (Figs. 3, 4). The wound track showed a direction approximately parallel to the horizontal plane of a neutral skull position.

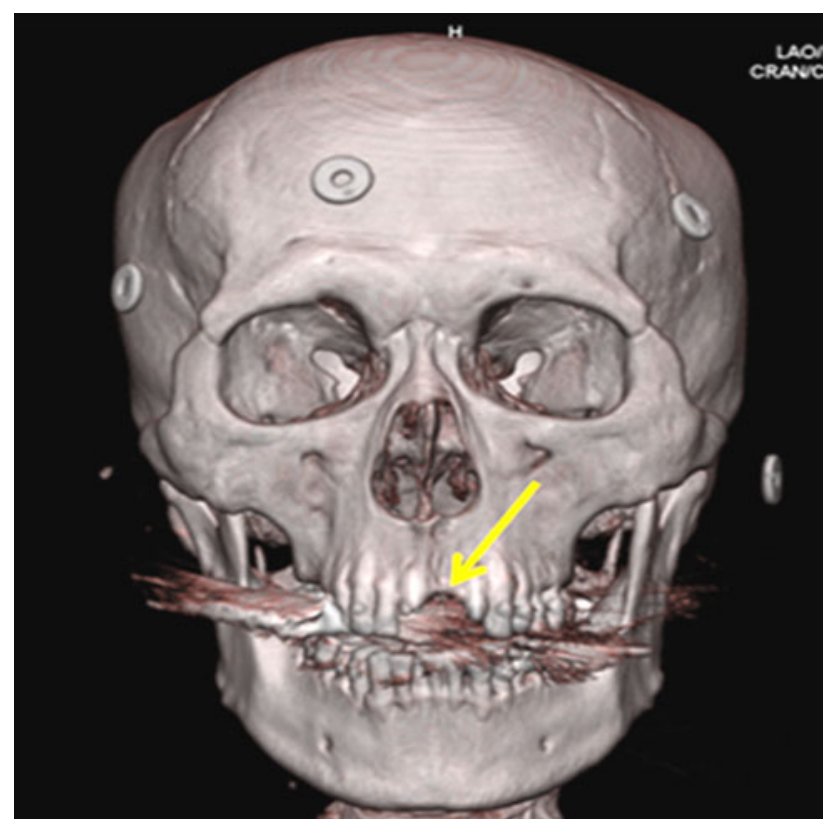

Fig. 1 CT, 3D reconstruction of the skull showing the destruction of the incisors (yellow arrow). Dental fillings created the streak artefacts. The ring-like structures are radiologic markers applied to the skin for a fusion of CT with surface scanning data

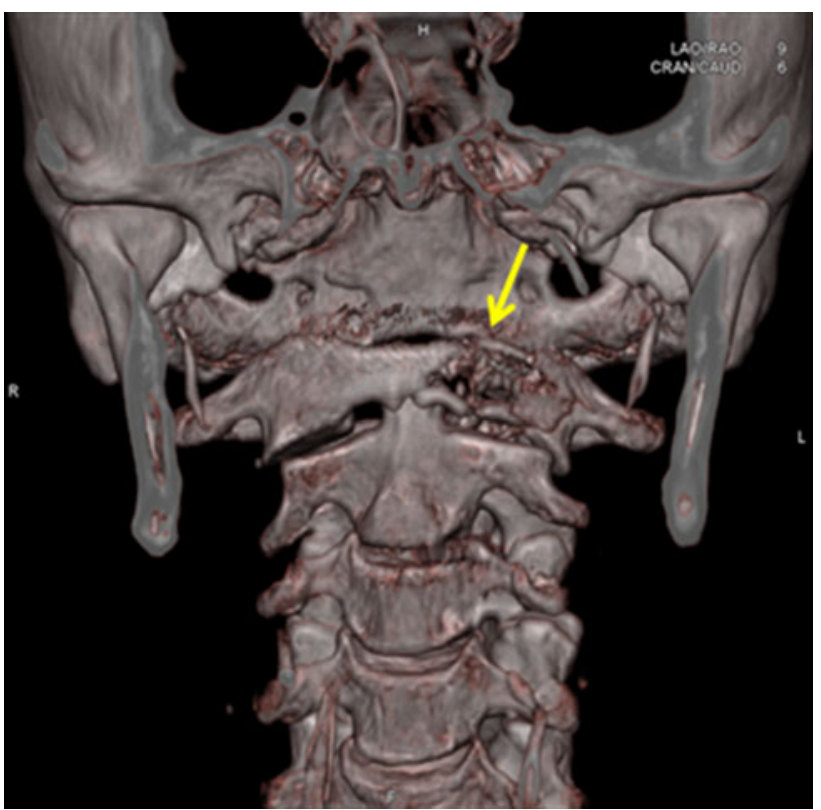

Fig. 2 CT, 3D reconstruction of the cervical spine after virtual removal of the frontal skull portion. The atlas is shot through on the left side (arrow)

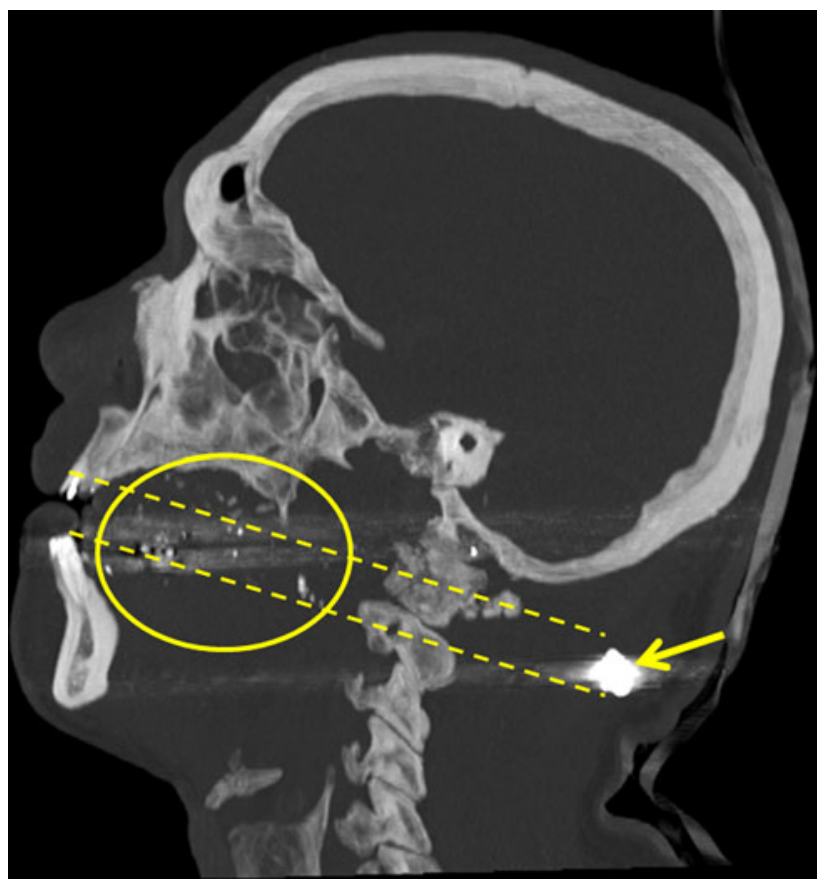

Fig. 3 CT, 2D sagittal reconstruction displaying bone and bullet fragments (encircled) along the bullet path (demarcated by dashed lines) to the nape, where the rest of the bullet is lodged (arrow)

\section{Autopsy}

Forensic autopsy was performed $19 \mathrm{~h}$ after the body was found. The mucosal lining of the upper lip presented an extensive tearing and hemorrhage. The lower lip displayed 


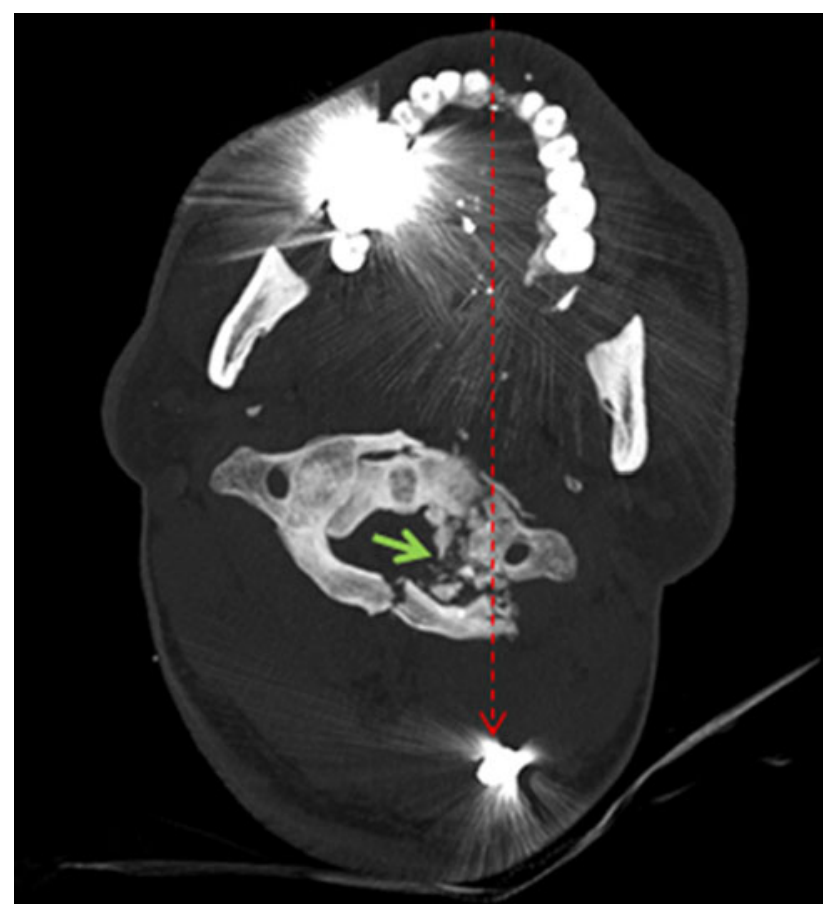

Fig. $4 \mathrm{CT}, 2 \mathrm{D}$ axial reconstruction displaying the approximate bullet course (red arrow) and the shot through atlas (green arrow)

mucosal hemorrhages mirroring the lower teeth (Fig. 5). The upper incisors (teeth 11 and 21) were broken out and missing and the tip of tooth 22 was broken. The floor of the mouth was severely injured and presented projectile fragments. A gunshot wound channel could be followed to the atlas, which displayed a fracture on the left side. The upper spinal cord was severely damaged. A slightly deformed full metal-jacketed projectile lay in the musculature of the nape of the neck on the left. There was a hematoma on the back of the head and a supra- and infratentorial subarachnoid hemorrhage.

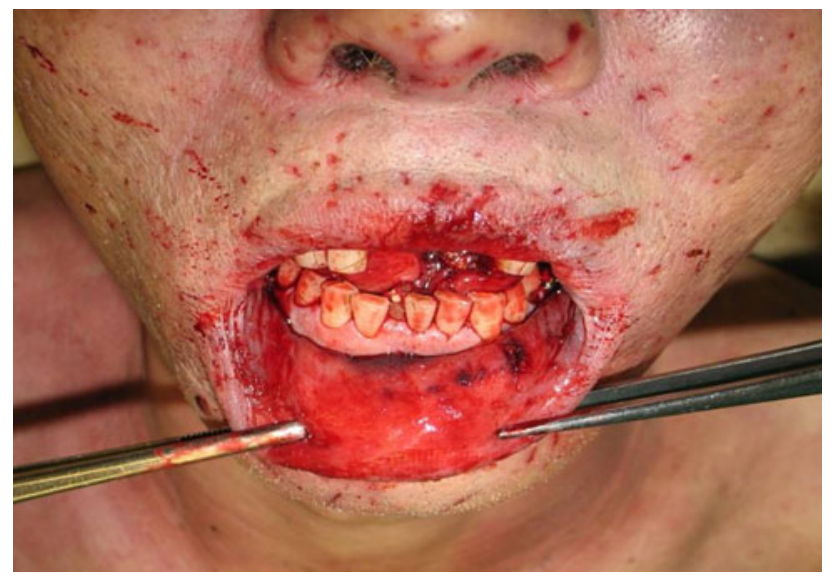

Fig. 5 Autopsy photograph showing the mucosal hemorrhages on the lower lip indicative of an externally induced pressure (i.e., from a muzzle) to the lips
Furthermore, aspirated gastric contents and blood as well as a discreet pulmonary fat embolism were noted.

Toxicological examination was negative for drugs and frequently abused medicaments in blood and urine. The alcohol-blood-concentration was $0.4 \mathrm{~g} / \mathrm{l}$ at the time of death.

Cause of death was a central respiratory paralysis due to traumatic (gunshot induced) medulla injury, the manner of death was deemed a homicide.

\section{Discussion}

Forensically relevant criteria to discriminate between suicide and homicide in intraoral gunshot fatalities are the site and the intraoral anatomic location of the entrance wound, the direction of the internal bullet path and the shooting distance [1-4].

Azmak [2] mentioned in his study on intraoral and perioral shooting cases that in all suicide cases shots were fired with the muzzle inserted into the oral cavity. In the homicide group of his study, seven out of eight cases had the entrance wound either on the corner of the mouth or on the outer surface of the lip(s). In only one of his investigated homicidal cases was the shot fired into the mouth.

In suicides, the victim usually points the muzzle upwards and the entrance wound is in the hard palate or posterior pharynx [2,4]. The direction of the bullet path in suicidal cases is mostly upward in relation to the horizontal plane $[1,2,4]$. In homicides, the entrance wound is commonly in the posterior pharynx and the tongue is also injured [2] and the bullet paths often appear to be directed parallel to the horizontal plane [1, 2, 4]. On the other hand, it was described by Zietlow [3], that there were three investigated homicidal intraoral gunshot wounds with an upward course through the palate.

As described before, PMCT is useful in determining a bullet course through a body [5-7]. In our case, PMCT clearly showed a wound track running approximately parallel to the horizontal plane in neutral position of the skull.

In contrast to suicidal gunshot activity, where the barrel of the gun is placed between the opened jaws, our case displayed a gunshot through the closed dentition. In addition, the lower lips showed hemorrhages consistent with marks from the lower canine teeth. In this case, it is assumed that, before shooting, the muzzle of the gun was pressed with force against the lips of the victim.

In conclusion, to determine the manner of death in cases of intraoral shooting fatalities an intensive medicolegal examination of the mouth, teeth and lips may be also helpful. In general, the finding of an intraoral gunshot wound should not only fix the mind on a suicidal incidence, 
but also include the rare possibility of a homicide, as seen in our presented case.

\section{Key points}

Several criteria help discriminate between a suicidal and homicidal intraoral gunshot:

(1) Firing distance; suicidal gunshots to the mouth are usually fired with the muzzle in the oral cavity, whereas homicidal gunshots may be fired outside the oral cavity, i.e., through the closed dentition.

(2) Site of entry wound; in suicides the entrance wound is often in the hard palate or posterior pharynx, in homicides commonly the entry often involves the posterior pharynx and tongue.

(3) Direction of the internal bullet path; the bullet path in suicidal cases is mostly upward in relation to the horizontal plane, as opposed to homicides, in which the path is more or less horizontal.

(4) Circumstances of death, especially the findings at the crime/incident scene.
Acknowledgments The authors thank Gary Hatch, MD, for assistance in manuscript preparation.

\section{References}

1. Karger B, Billeb E, Koops E, Brinkmann B. Autopsy features relevant for discrimination between suicidal and homicidal gunshot injuries. Int J Legal Med. 2002;116:273-8.

2. Azmak D, Altun G, Koç S, Yorulmaz C, Özaslan A. Intra- and perioral shooting fatalities. Forensic Sci Int. 1999;101:217-27.

3. Zietlow C, Hawley DA. Unexpectedly homicide; three intraoral gunshot wounds. Am J Forensic Med Pathol. 1993;14:230-3.

4. Fatteh A. Homicidal gunshot wound of mouth. J Forensic Sci Soc. 1972;12:347-9.

5. Thali MJ, Schweitzer W, Yen K, et al. New horizons in forensic radiology: the 60-second digital autopsy-full-body examination of a gunshot victim by multislice computed tomography. Am J Forensic Med Pathol. 2003;24:22-7.

6. Thali MJ, Yen K, Vock P, et al. Image-guided virtual autopsy findings of gunshot victims performed with multi-slice computed tomography and magnetic resonance imaging and subsequent correlation between radiology and autopsy findings. Forensic Sci Int. 2003;138:8-16.

7. Andenmatten MA, Thali MJ, Kneubuehl BP, et al. Gunshot injuries detected by post-mortem multislice computed tomography (MSCT): a feasibility study. Leg Med (Tokyo). 2008;10:287-92. 